\title{
El aporte de "Lo Santo" a la comprensión de la experiencia religiosa: Una mirada desde la Psicologia de la Religión
}

\author{
The contribution of "The Holy" to the understanding of the religious experience: \\ A view from the Psychology of Religion
}

Patricia Pizzorno*

\begin{abstract}
Resumen
Desde el ángulo específico de la psicología de la religión, el presente artículo pretende dar cuenta del aporte de Rudolf Otto en "Das Heilige" a la comprensión de la experiencia religiosa. Tomando como punto de partida el abordaje de las ciencias religiosas en donde Otto ha marcado un hito fundamental, se realiza a continuación un recorrido por autores que se ocuparon en forma positiva de la experiencia religiosa en el ámbito de la psicología. El artículo concluye dejando una puerta abierta hacia la investigación de la experiencia religiosa en el campo de las neurociencias de la religión.
\end{abstract}

\section{Palabras clave}

Religión. Psicología. Experiencia religiosa. Rudolf Otto.

\begin{abstract}
From the specific angle of the psychology of religion, the present article tries to give an account of the contribution of Rudolf Otto in "Das Heilige" to the understanding of the religious experience. Taking as a starting point the approach of the religious sciences where Otto has marked a fundamental milestone, a tour is then made by authors who deal positively with the religious experience in the field of psychology. The article concludes by leaving an open door to the investigation of religious experience in the field of neurosciences of religion.
\end{abstract}

\section{Keywords}

Religion. Psychology. Religious experience. Rudolf Otto.

[Texto recebido em janeiro de 2018 e aceito em janeiro de 2018, com base na avaliação cega por pares realizada por pareceristas ad hoc]

* Licenciada en Psicología (Universidad de la República en Montevideo, Uruguay). Master en Teología Bíblica (Facultades EST de Sao Leopoldo, Brasil). Master en Derecho Canónico (Pontificia Universidad Gregoriana de Roma, Italia). Magister en Ciencias Religiosas (Pontificia Universidad Gregoriana de Roma, Italia). Profesora titular de Psicología de la Religión en la Universidad Católica del Uruguay, en Montevideo, Uruguay. E-mail: patricia_pizzorno@yahoo.it 


\section{Introducción}

El fenómeno de las creencias religiosas necesita ser explicado, siendo que la religión es un fenómeno humano universal. En la mayoría de las culturas humanas hay gente que cree que hay algo más allá de la muerte, que los rituales pueden cambiar las cosas, y que la enfermedad y la desgracia son causadas y/o aliviadas por entidades invisibles. Este fenómeno aparentemente irracional se ha mantenido vivo en el tiempo, lo que constituye un enigma para el psicólogo.

A lo largo del tiempo el fenómeno religioso ha suscitado las más diversas reacciones en un abanico que se abre 360 grados y abarca infinitas gradaciones desde la total y ferviente adhesión de fe al rechazo rotundo, pasando tanto por el cálido asombro y la curiosidad deseante como por la distante indiferencia. Al interno de lo que podríamos llamar el fenómeno religioso, la experiencia religiosa ocupa un lugar privilegiado tanto en la vida cotidiana de gran cantidad de personas de diversos orígenes y culturas, como en el debate científico. La experiencia religiosa, en sus múltiples variantes, forma parte de la intimidad de muchos seres humanos a lo largo y ancho del planeta y a lo largo de la historia ha sido canal y testigo de profundas emociones humanas. Un estudio demográfico global de más de 230 países y territorios, basado en el análisis de más de 2.500 censos, encuestas y registros de población, y llevado a cabo por el Pew Research Center en 2012,1 estima que en el mundo hay 5.8 mil millones de niños y adultos que poseen una afiliación religiosa, lo que representa el 84\% de la población del mundo que en el 2010 era de 6,9 mil millones. Un siguiente estudio realizado por el Foro encontró que aproximadamente una de cada seis personas en todo el mundo (el 16\%) no tiene ninguna afiliación religiosa. Pero las encuestas indican que muchos de los no afiliados poseen creencias religiosas o espirituales (como la creencia en Dios o en un espíritu universal) a pesar de que no se identifiquen con una fe en particular. ¿Pero cuál es la naturaleza de la experiencia religiosa? ¿Es una experiencia religiosa en sí, original, sui generis según la definición de Ann Taves para quien la experiencia sui generis es una experiencia única según la expresión latina que significa "de su propia especie" ${ }^{2}{ }^{2} \mathrm{O}$ es producto del contexto personal y social? ¿Existe en el cerebro un correlato neural de la experiencia religiosa? El aporte de Rudolf Otto es, en este contexto, fundamental para la comprensión de la experiencia religiosa, especialmente la experiencia sui generis que encuentra en él uno de sus primeros y principales exponentes.

Esbozado el amplio horizonte del fenómeno religioso y en él, de la experiencia religiosa, se clarificarán términos importantes. A continuación, se precisará qué entienden por experiencia religiosa algunos referentes clásicos del ámbito de la fenomenología y la

1 THE GLOBAL RELIGIOUS LANDSCAPE. Pew Research Center. (2012). Disponible en: <http://www.pewforum.org/2012/12/18/global-religious-landscape-exec>. Recuperado 22 ene. 2017.

2 TAVES, Ann. Religious Experience Reconsidered: A Building-Block Approach to the Study of Religion and Other Special Things. Princeton and Oxford: Princeton University Press, 2009. 
historia comparada de las religiones y se profundizará en el aporte de Otto. Luego se abordará el campo de la psicología de la religión desde la postura de algunos referentes clásicos y para concluir se esbozarán las principales líneas de investigación sobre la experiencia religiosa en el área las neurociencias, dejando abierta la puerta a una próxima profundización en este ámbito. Se concluirá con las reflexiones personales y el esbozo del camino a seguir.

\section{Definiendo términos Religión}

A lo largo de la historia el término religión ha tenido gran variedad de significados y definiciones ya que engloba una inmensa gama de realidades muy distintas, personales, institucionales, culturales, de pensamientos, sentimientos y prácticas, y es un hecho social total que abarca todas las dimensiones del vivir humano: las relaciones interpersonales, la organización social, el arte, la sexualidad, la economía, el lenguaje, los lazos familiares. ${ }^{3}$ Esa amplitud del fenómeno religioso hace muy difícil su definición hasta el punto de que no haya una definición en la que al menos una mayoría concuerde. Incluso hay estudiosos que dudan de que se pueda llegar a formular una. ¿Religión es el sentido de lo divino de Max Müller, concebido en términos de infinito en su Mitología comparada de 1856? ¿Es la experiencia de lo sagrado como sostiene Rudolf Otto en Lo Santo publicado en 1917? ¿O la creencia en seres espirituales como propone E. B. Tylor ${ }^{4}$ en 1871 en Cultura primitiva, para quien el animismo, fundamento de toda religión, consiste en la creencia en espíritus que habitan y animan seres, o almas que existen en cosas? ¿O la distinción entre lo sagrado y lo profano que propone Mircea Eliade en Lo sagrado y lo profano en $1967 ?$

Para la Real Academia Española ${ }^{5}$ la religión es a nivel cognitivo, un conjunto de creencias y dogmas sobre la divinidad; a nivel de las emociones, un conjunto de sentimientos de veneración y temor hacia esta divinidad; y a nivel de la praxis, el conjunto de normas morales para la conducta tanto individual como social, y las prácticas rituales con las cuales se le rinde culto. El término religión proviene del latín religĭo, -ōnis y etimológicamente podría tener dos explicaciones. ${ }^{6}$ Para Cicerón (106-43 a. C.), en De natura deorum, 7 II, 28, escrito 45 a.C., el sustantivo religio se deriva del verbo relegere o religere que aplicados al culto significan observancia cuidadosa. Lactancio (250-325 d.C.) por su parte,

3 ALETTI, Mario. Percorsi di Psicologia della Religione alla luce della Psicoanalisi. 2.ed. Roma: Aracne, 2010.

4 STRENSKI, I. The Shock of the «Savage»: Edward Burnett Tylor, Evolution, and Spirits. Thinking About Religion: An Historical Introduction to Theories of Religion. Oxford: Blackwell Publishing Ltd., 2006.

5 REAL ACADEMIA ESPAÑOLA. Diccionario de la Lengua Española. 23.ed. Madrid: 2014. Disponible en: <http://www.rae.es/diccionario-de-la-lengua-espanola/la-23a-edicion-2014>.

6 LEWIS, C. T.; SHORT, C. Latin Dictionary. Oxford: Clarendon Press, 1879.

7 CICERÓN. De natura deorum. Libro II, 28. Disponible en: <http://www.progettovidio.it/dettagli1.asp?id=5519\&opera=De\%20Natura\%20Deorum\&libro=Libro\%2 0II>. Recuperado 5 feb. 2017. 
en Divinae Institutiones IV,8 28, redactadas entre el 304-313 d.C., hace derivar la palabra religio del verbo latino religare que significa vincular, religar, atar fuertemente. Es así como para Cicerón la religión es un asunto de culto cuyas reglas hay que observar fielmente y para Lactancio ésta tiene un carácter más existencial de religación del hombre con la trascendencia.

Quizás de estas dos posibles etimologías se puedan deducir dos modalidades de comportamiento religioso descritos por la mayoría de los estudiosos desde el punto de vista de la psicología de la religión. Según la etimología derivada de Cicerón, la religiosidad de los individuos sería más bien una realidad extrínseca ligada a la observancia de reglas cultuales, mientras que para la derivada de Lactancio sería sobre todo una realidad intrínseca ligada a una ligación/relación personal con el Trascendente. William James hace una diferencia entre quienes viven la religión como "una fiebre aguda" y quienes la viven como una "costumbre sin vida". 9 Y como él varios autores diferencian dos modos, o incluso tres, de ser religioso. Sin pretender ser exhaustivos, se presentan algunos en la tabla 1.

Tabla 1: Modalidades de religiosidad

\begin{tabular}{ccc}
\hline MODO 1 & MODO 2 & AUTOR \\
\hline Observancia cuidadosa & Atar fuertemente & $\begin{array}{c}\text { Lactancio (Divinae } \\
\text { Institutiones IV, 28) }\end{array}$ \\
\hline Cristianismo Oficial & Cristianismo Radical & Cicerón (De natura deorum, II, \\
Costumbre sin vida & Fiebre aguda & Kirkegaard10 \\
Neutralizada & James \\
\hline Fe dogmática & Experiencia de fe personal & Adorno \\
Inmadura & Fe reflexionante & Kant ${ }^{11}$ \\
Extrínseca & Madura & Allport12 \\
Utilitaria & Intrínseca & Allport y Ross ${ }^{13}$ \\
\hline
\end{tabular}

LACTANCIO. Divinae Institutiones. v. IV, 28. Madrid: Gredos, 1990.

9 JAMES, William. Las variedades de la experiencia religiosa: Un estudio de la naturaleza humana. Madrid: Península, 1994. p. 7.

10 DRU, A. The Journals of Søren Kierkegaard. Oxford: University Press, 1938.

11 KANT, I. La religión dentro de los límites de la mera razón. Madrid: Alianza Editorial, 1969.

12 ALLPORT, G. The individual and his religion: a psychological interpretation. London: Constable Publications, 1950.

13 ALLPORT, G.; ROSS, J. M. Personal religious orientation and prejudice. Journal of Personality and Social Psychology, v. 5, p. 432-443, 1967.

14 BATSON, D. Religion and the Individual. New York: Oxford University Press, 1982. 


\begin{tabular}{ccc}
\hline Autoritaria & Humanística & Fromm ${ }^{15}$ \\
$\mathbf{R}$ (Religión con mayúscula) & $\mathrm{r}$ (religión con minúscula) & Maslow $^{16}$ \\
Consensual & Comprometida & Spilka $^{17}$ \\
\hline
\end{tabular}

En las ciencias sociales encontramos también dos modalidades de definición de la religión. Una sustancialista y otra funcionalista. La religión sustancialista está vinculada a autores clásicos como Durkheim, Weber y Marx. Sigue el modelo de las religiones históricas, y tiene una estructura organizada institucionalmente con un conjunto de creencias, dogmas y ritos. La religión funcionalista está vinculada a autores como Parsons, Malinowski, Luckmann y Berger. Se pregunta el para qué de la religión y la define como una construcción imaginaria, amplia e inclusionista, relacionada con la búsqueda de sentido y la trascendencia. Incluye las diversas prácticas religiosas vinculadas a la modernidad y la posmodernidad. ${ }^{18}$

\section{Espiritualidad}

El término espiritualidad viene definido por la Real Academia Española en base al término espíritu. Este a su vez proviene del latín spiritus que significa soplo de aire, hálito, aliento; pero también inspirar y espirar (respirar) e incluso espirar el espíritu, es decir morir. El vocablo spiritus proviene del verbo spirare, que suena casi como el rumor del aire que se espira y probablemente tiene un origen onomatopéyico. Pero así como el término español espíritu traduce al latín spiritus, traduce también con diferentes matices y acepciones al hebreo ruah que significa literalmente viento, al sánscrito Ātman usado en las religiones de la India y al griego pneuma.

En una conferencia pronunciada a la Sociedad Literaria de Augsburgo el 20 de octubre de 1926 sobre la naturaleza y el espíritu, C. G. Jung expresaba que espíritu y vida son términos antiguos y muy conocidos que han sido relacionados y combinados de diversas maneras a lo largo de miles de años. Según Jung en los albores del tiempo "alguien descubrió que el aliento vivo que abandona el cuerpo del hombre que muere significaba más que aire en movimiento", y por ende, no puede ser casualidad que

\footnotetext{
FROMM, E. Psicoanálisis y religión. Buenos Aires: Editorial Psique, 1956.

MASLOW, A. Religions, values and peak-experiences. London: Penguin Books, 1994.

17 SPILKA, B.; SHAVER, P.; KIRKPATRICK, L. A. A general Attribution Theory for the Psychology of Religion. Journal for the Scientific Study of Religion, v. 24, n. 1, p. 1-20, 1985; HOOD, R.; SPILKA, B.; HILL, P. C. The Psychology of Religion: An empirical approach. 4.ed. New York: The Guilford Press, 1996.

18 APUD, Ismael. Magia, ciencia y religión en antropología social. De Tylor a Levi-Strauss. Revista Crítica de Ciencias Sociales y Jurídicas, v. 30, n. 2, 2011.
} 
palabras onomatopéyicas como ruaj, ruch y roho en hebreo, árabe y swahili respectivamente, signifiquen "espíritu" al igual que el griego pneuma y el latín spiritus" ${ }^{19}$

De este breve recorrido etimológico podría inferirse que espiritualidad es un modo de vivir dando espacio a esa condición del espíritu como aliento vital en cada ser humano. Es algo más amplio, más libre, más abierto y universal que la dimensión religiosa. Esa dimensión de la existencia, personal y a la vez universal la he llamado en la figura 1 que sigue a continuación, espiritualidad “e”, y representa el círculo exterior, más grande y más abierto. Al interno de esa espiritualidad " $\mathrm{e}$ " encontramos un círculo intermedio que es el de la Religión (" $R$ ") con todas sus posibles facetas tanto institucionales como personales. Dentro de este segundo círculo hay otro más pequeño que es el de las diferentes confesiones religiosas expresado con la letra " $\mathrm{C}$ ", y aún dentro de este, la espiritualidad “ $E$ " que es la manera distintiva personal o grupal, en la que se puede vivir la religión al interno de las diferentes confesiones religiosas. Al interno del cristianismo encontramos diversas corrientes de espiritualidad, modos variados de vivir el mismo Evangelio desarrollados en la historia de las diferentes Iglesias y en la inculturación de las diferentes corrientes litúrgicas y teológicas en los diversos contextos. En el mundo islámico encontramos divisiones como la sunita y la chiita, que, aunque son de carácter social y político, representan diferentes maneras de vivir la adhesión al Corán. También tenemos la espiritualidad sufí, que representa su aspecto místico. La rama sunita tiene cuatro escuelas de interpretación de la jurisprudencia islámica que se expresan en diferentes modos de vivir. ${ }^{20}$ Esos diferentes modos de vivir conforman diferentes espiritualidades ("E"). Si bien el término espiritualidad pertenece al hebreo moderno y la espiritualidad en el judaísmo nace en la modernidad, también allí podemos encontrar diferentes modos y corrientes de vivir la religión al interno del gran universo de la religión judía.

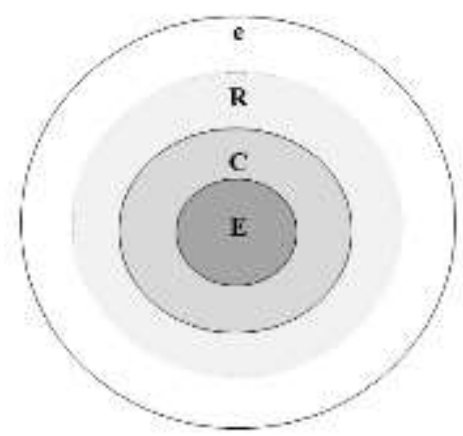

Figura 1: Relación entre religión (R), confesión religiosa (C) y espiritualidad (e/E)

En el ámbito de la Psicología de la Religión ha habido una búsqueda de definir y de relacionar los campos de la religión y la espiritualidad, debate que Aletti ubica en estos

19 JUNG, Carl G. Structure and dynamics of the psyche. v. 8. 2.ed. Princeton: Bollingen Series, 1969. p. 319.

20 STEWART, D. J. The structure of the fihrist: Ibn Al-Nadim as historian of Islamic legal and theological schools. International Journal of Middle East Studies, v. 39, p. 369-387, 2007. 
últimos 10 años y que según él ha dado origen a diversas posturas. ${ }^{21}$ Hay quienes apoyan la intersección parcial, otros la superposición de ambas, y otros incluso llegan a afirmar la total identidad entre una y otra. Algunos autores sostienen la inclusión de la espiritualidad en la religiosidad, y otros lo contrario. Tanto es así que algunos autores debaten si la disciplina debería ser Psicología de la Religión, Psicología de la Espiritualidad, o Psicología de la Religión y Espiritualidad. De hecho, este último es el nombre que toma actualmente la división 36 de la Asociación Psicológica Americana (APA). El esquema sugerido en la Figura 1 es otra propuesta que podría dar cuenta de la relación entre religión y espiritualidad.

\section{Psicología de la Religión y la Espiritualidad}

Es la rama de la psicología que se aventura a estudiar la conducta religiosa, la actitud del ser humano frente al hecho religioso. Es el estudio, con métodos e instrumentos psicológicos, de aquello que de psíquico hay en la religión. Se basa en el estudio positivo del fenómeno religioso como conducta observable, tipificable y cuantificable según los modelos teóricos de las ciencias empíricas. Como principio epistemológico excluye la pretensión de negar o probar, y busca contribuir a la comprensión del fenómeno religioso sin pronunciar juicios sobre la realidad ontológica del polo objetual de la Religión. Según la mencionada división 36 de la APA:

La Asociación para la Psicología de la Religión y la Espiritualidad promueve la aplicación de métodos de investigación psicológica y marcos interpretativos a diversas formas de religión y de espiritualidad; fomenta la incorporación de los resultados de este trabajo en la clínica y en otros encuadres; y promueve el diálogo constructivo y el intercambio entre el estudio y la práctica psicológica por un lado, y entre perspectivas e instituciones religiosas por el otro. La división es estrictamente no partidaria y acoge la participación de todas las personas que ven a la religión como un factor significativo de la actividad humana. ${ }^{22}$

\section{Fenomenología de la religión}

La fenomenología de la religión es un saber descriptivo que busca comprender la estructura significativa del hecho religioso en la amplia variedad de sus formas, e indaga su significado por la referencia al contexto vivido y a la experiencia que expresa. En el origen del término fenomenología encontramos dos tradiciones filosóficas diferentes. Una de origen alemán, privilegia la concepción de fenomenología como el método propio de

21 ALETTI, 2010, p. 37.

22 ASOCIACIÓN PSICOLÓGICA AMERICANA. Asociación para la Psicología de la Religión y la Espiritualidad, División 36. Disponibel en: <http://www.apa.org/about/division/div36.aspx>. Recuperado 4 feb. 2017. 
una filosofía rigurosa. Esta tradición a la que pertenece Hegel, encuentra su mayor exponente en Husserl y sus discípulos, entre los que se encuentran Max Scheler y Edith Stein. La otra tradición es de origen anglosajón y es característica de la historia comparada de las religiones. Está representada por autores como Rudolf Otto y Mircea Eliade. Esta modalidad privilegia la observación y descripción sistemática de los hechos religiosos y la comparación de los datos que emergen de dicha descripción. Posee como rasgos característicos el estudio del fenómeno religioso en lo que éste tiene de específicamente religioso, da especial importancia a la intencionalidad que lo anima y le da significado, y se mantiene neutral con respecto al objeto del fenómeno religioso sin decidir sobre su veracidad.

Según Velasco, ${ }^{23}$ de la comparación de los datos que surgen de la observación de los hechos religiosos emerge una estructura significativa con rasgos comunes. Estos son: el sentido de lo sagrado (entendido como opuesto a lo profano según el planteo de Eliade ${ }^{24}$ ); la presencia del misterio por el cual los hombres religiosos han realizado ritos, ceremonias, oraciones, bailes o sacrificios; y algún tipo de experiencia supuesta por o a través de esas acciones. Taves sintetiza más estos rasgos comunes y habla solamente de un núcleo común que apunta a lo trascendente ${ }^{25}$ y Uffe Schjoed ${ }^{26}$ sostiene que, aunque el comportamiento religioso abarca pensamientos y prácticas muy diferentes y muchas probabilidades de diferir tanto en el contenido cognitivo como en los correspondientes correlatos neurales, existe una característica compartida por todos estos fenómenos, que es el vínculo a una instancia sobrenatural.

\section{La experiencia religiosa}

La palabra experiencia viene definida por la Real Academia Española como el "hecho de haber sentido, conocido o presenciado alguien algo", la "práctica prolongada que proporciona conocimiento o habilidad para hacer algo", el "conocimiento de la vida adquirido por las circunstancias o situaciones vividas" y por último una determinada "circunstancia o acontecimiento vivido por una persona". Si a experiencia le anexamos el adjetivo religiosa, encontramos que la expresión describe la dimensión subjetiva de la religión enfatizando la experiencia personal emocional y una forma intuitiva de conocimiento en la que el individuo funda su certeza religiosa. Vergote ${ }^{27}$ haciendo uso de la expresión acuñada por Otto, designa la experiencia religiosa como "la captación, en lo que es humano y terrestre, del impacto de lo Totalmente-Otro" y sostiene que es la misma

23 VELASCO, J. M. Introducción a la Fenomenología de la Religión. 7.ed. (corregida y ampliada). Madrid: Cristiandad, 1978; VELASCO, J. M. Religión. Disponible en: <ttp:/ / www.mercaba.org/DicPC/R/religion.htm>. Recuperado 3 feb. 2017.

24 ELIADE, M. Historia de las ideas y las creencias religiosas. v. I. Madrid: Ediciones Cristiandad, 1978.

25 TAVES, A. Religious Experience. Encyclopedia of Religion. 2.ed. 2005. p. 7736-7750.

26 SCHJOEDT, U. The Religious Brain: A General Introduction to the Experimental Neuroscience of Religion. Method and Theory in the Study of Religion, v. 21, p. 310-339, 2009.

27 VERGOTE, Antoine. Psicología religiosa. Madrid: Taurus, 1966. p. 24. 
experiencia humana óptima que toma sentido por el contexto y por el lenguaje. Se caracteriza por la subjetividad y por el contexto del individuo, y se encuentra en el cruce del horizonte histórico-cultural y del desarrollo personal, individual, identitario (Figura 2).

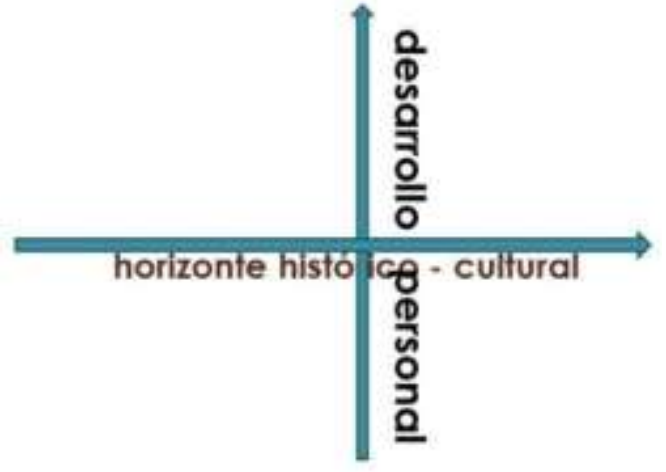

Figura 2: Dimensiones de la Experiencia Religiosa

La amplitud del fenómeno religioso y la dificultad de definición y de sistematización de los conceptos, hacen difícil el estudio cognitivo y neuropsicológico de la experiencia religiosa. Según Czachesz, ${ }^{28}$ los estudios han utilizado diferentes nociones de experiencia originadas a partir de disciplinas y contextos diversos, lo que dificulta la comparación tanto de los paradigmas de investigación como de los resultados obtenidos. Los contextos desde donde ha sido abordado el estudio de la experiencia religiosa se pueden expresar como muestra la Figura 3. Es claro que todas las áreas aportan a la comprensión de la experiencia religiosa, pero también es evidente la necesidad de un estudio interdisciplinar.

\section{El aporte de las ciencias de la religión}

La primera noción de la experiencia religiosa proviene de las ciencias de la religión. En el origen de las grandes religiones se encuentran numerosos relatos de experiencias religiosas. La Torá y la Biblia narran experiencias de diversa índole en patriarcas, profetas y apóstoles y la tradición islámica cuenta las experiencias de Mahoma. Dios bendice a Adán y Eva en el paraíso y a cada uno de los patriarcas. Abraham habla con Dios como con un amigo, Jacob lucha con un ángel en Penuel y a Moisés Dios le habla desde una zarza ardiente. Isaías viaja al cielo así como Pablo de Tarso, y Mahoma encuentra un arcángel en la cueva de Hira. Los Evangelios relatan las experiencias religiosas de Jesús entre las que se encuentra el bautismo, la experiencia mística de transfiguración y la intensa oración del Getsemaní antes de ser ajusticiado. Narran también las experiencias de sus discípulos. Las experiencias vocacionales de los profetas

28 CZACHESZ, I. Jesus' religious experience in the Gospels: toward a cognitive neuroscience approach. En: Jesus - Gestalt und Gestaltungen. Rezeptionen des Galiläers in Wissenschaft, Kirche und Gesellschaft. Göttingen: Vandenhoeck \& Ruprecht, 2013. p. 553-580; CZACHESZ, I. Tours of heaven in light of the neuroscientific study of religious experience. Journal of Cognitive Historiography, in press, v. 2, p. 33-55, 2015. 
en el Antiguo Testamento y de los discípulos en el Nuevo, son auténticas experiencias religiosas. Los relatos son numerosos y partiendo desde entonces hasta hoy, describen una amplia diversidad de experiencias subjetivas sobre las cuales, al decir de Czachesz, hay un vacío en lo que respecta a su estudio científico y sistemático. Pero ese vacío comienza a llenarse a partir de la fenomenología de la religión ligada principalmente al protestantismo alemán, desde donde se considera la experiencia religiosa como un tipo especial de experiencia, no mediada, y descrita como un encuentro con la trascendencia, lo sagrado, lo numinoso, el misterio. Son experiencias sui generis a las que se refieren estudiosos como Friedrich Schleiermacher, Rudolf Otto, Mircea Eliade, y Joachim Wach. En Lo Santo, Rudolf Otto (2001 [1917]) se aventura a describirla. Partiendo del sentimiento religioso de dependencia de Schleiermacher al que Otto denomina sentimiento de criatura, el autor afirma que este es concomitante a un objeto fuera del sujeto y al que llama con un término acuñado por él mismo, lo numinoso, el mysterium tremendum y fascinans. ${ }^{29} \mathrm{La}$ experiencia religiosa así concebida es entendida como una experiencia sui generis, única en su especie, original, totalizante y totalizadora en la vida del sujeto, intransferible, inefable, e inenarrable. Otto es de los primeros autores (¡si no el primero!) en intentar describirla y tanto su descripción como el vocabulario que en forma original y creativa emplea para hacerlo, serán referencia para tantos autores que, desde las más diversas áreas, intenten detrás de él acercarse a la comprensión de esta experiencia sublime. La experiencia religiosa es difícil deponer en palabras. Sin embargo se pueden encontrar descripciones lingüísticas de estas experiencias inenarrables y a través de su análisis se pueden descubrir características subyacentes y comunes. Del estudio realizado por un grupo de psicólogos de la religión en diversas universidades de Estados Unidos sobre una muestra de 777 narraciones escritas, surge que las personas que tienen experiencias místicas supuestamente inefables e indescriptibles, las describen utilizando un lenguaje más inclusivo y menos palabras religiosas que las personas que no las tienen, y que estas experiencias producen en ellas un impacto profundamente positivo. ${ }^{30} \mathrm{El} \mathrm{mismo} \mathrm{Otto} \mathrm{no}$ encuentra en el vocabulario religioso tradicional un término adecuado y recurre a términos originales para poder describirla. Términos como lo numinoso, y expresiones latinas que no se traducen, que entran a formar parte del vocabulario místico.

\section{El aporte de la psicología}

En ámbito psicológico e influenciados por la teología de las experiencias sui generis y recurriendo en ocasiones a las mismas expresiones de Otto, William James, Carl G. Jung y Abraham Maslow entienden la experiencia religiosa respectivamente como una fiebre

29 OTTO, R. Lo Santo: Lo racional y lo irracional en la idea de Dios. Madrid: Alianza Editorial, 2001. p. 17-20.

30 YADEN, D.; EICHSTAEDT, J.; SCHWARTZ, A.; KERN, M.; NGUYEN, K. L.; WINTERING, N.; NEWBERG, A. The language of ineffability: linguistic analysis of mystical experiences. Psychology of Religion and Spirituality, 2015. 
aguda, una experiencia personal de lo numinoso de la cual el individuo es víctima, y una experiencia cumbre en la vida del sujeto.

En 1902 James escribe Las variedades de la experiencia religiosa, un estudio de la naturaleza humana, que según el prefacio del autor es descriptivo de las "necesidades religiosas del hombre". ${ }^{31} \mathrm{Su}$ primera conferencia se tituló "Religión y Neurología" pero James nunca pudo desarrollar una neurología de la experiencia religiosa, ya que en ese entonces ésta estaba recién emergiendo como ciencia y faltaban las bases científicas y metodológicas para una verdadera ciencia de la religión. En la tercer conferencia titulada "La realidad de lo no visible", James afirma que la vida de la religión (así es como él llama a la religiosidad) "consiste en creer en un orden no visible y que nuestra felicidad estriba en ajustarnos armoniosamente a él". ${ }^{32}$ Sostiene también la normalidad de la conducta religiosa que considera una característica típica del comportamiento humano. Esta conducta religiosa es la expresión de los sentimientos que nacen de la experiencia religiosa personal y que describe como: "los sentimientos, los actos, las experiencias de los individuos en la soledad de su alma, en cuanto se sienten en relación con aquella cosa que ellos puedan considerar como divina". ${ }^{33}$ La religión para James es algo interior, personal y subjetivo, que se distingue de la institución. Para analizar la experiencia religiosa James tomará en cuenta principalmente a las personas para las cuales la religión "es una fiebre aguda" y no a aquellas para quienes la religión es simplemente una "conducta imitativa" pues se deben buscar las "experiencias originales". ${ }^{34}$ Las personas que experimentan esta fiebre aguda son líderes religiosos a quienes el autor llama genios en su ámbito. Son personas que, según se desprende de sus propias narraciones, han vivido una experiencia sui generis como resulta de los relatos que James presenta en sus conferencias. James fue un pionero en el estudio del fenómeno religioso desde el punto de vista psicológico investigando las bases neurológicas de la experiencia religiosa.

Para Jung la religión es la actitud de una conciencia que ha sido transformada por la experiencia personal de lo numinoso. La religión no se prueba: se experimenta y se impone ya que para él es una función psíquica irreductible. En 1949 en Psicología y Religión, Jung explica qué entiende por el término religión haciendo referencia al numinoso de Rudolf Otto al que da un toque personal: “una existencia o efecto dinámicos no causados por un acto arbitrario, sino que, por el contrario, el efecto se apodera y domina al sujeto humano que siempre, más que su creador, es su víctima". ${ }^{35}$ Para Jung, lo numinoso es una condición del sujeto, independiente de su voluntad, y la religión es "una actitud especial del espíritu humano", "la particular actitud de una conciencia transformada por la

31 JAMES, 1994, p. 4.

32 JAMES, 1994, p. 28.

33 JAMES, 1994, p. 18.

34 JAMES, 1994, p. 7.

35 JUNG, Carl G. Psicología y religión. Buenos Aires: Paidós, 1949. p. 8. 
experiencia de lo numinoso. ${ }^{36}$ Para referirse a ella, agrega en Respuesta a Job, que al tener que hablar de "factores numinosos" necesita usar no sólo su entendimiento sino también su sentimiento, su "subjetividad emocional".37

En Religiones, valores y experiencias cumbre, Maslow describe lo que llama experiencias cumbre y según afirma se pueden dar tanto en ámbito religioso como en ámbito secular. La prueba de esto es que términos como sagrado, divino, santo, numen, salvación y reverencia, entre otros, han sido utilizados para describir experiencias cumbre indistintamente en sentido natural y sobrenatural. A la hora de definir la religión, Maslow afirma que existen dos tipos. Una que es organizada e institucionalizada y la caracteriza con la " $R$ ", y otra que es naturalista y subjetiva y la caracteriza con la "r". ${ }^{38} \mathrm{Y}$ afirma que muchas veces la Religión es la mayor enemiga de la experiencia religiosa. Maslow sostiene que el núcleo intrínseco y la esencia de las religiones reveladas ha sido la iluminación o revelación personal y privada, la experiencia del éxtasis de algún profeta. Por eso estas religiones se autodenominan religiones reveladas, se fundan en la iluminación en la que encuentran su fuente, y tienen su derecho a existir en la codificación y comunicación de esta iluminación a los demás. Estas iluminaciones místicas son experiencias cumbre. Y la experiencia cumbre religiosa de Maslow es una experiencia sui generis. Maslow afirma haber encontrado ese mismo tipo de experiencias, y en ocasiones descriptas en un lenguaje similar, en ámbito laico. Para él la experiencia cumbre puede ser tanto religiosa como secular, y la diferencia entre unas y otras está en el modo en que el sujeto las considera. Es decir que una experiencia cumbre puede ser o no ser considerada una experiencia religiosa, según el sentido y contenido que le atribuye el sujeto que la vivencia.

Pero la noción de experiencia sui generis no es la única en ámbito psicológico. Gordon Allport, Erich Fromm y Víctor Frankl se ocupan del fenómeno religioso en una línea más abarcativa sin hacer referencia a las grandes experiencias sui generis. En El individuo y su religión, Allport (1950) analiza la religiosidad de las personas maduras. Describe la experiencia religiosa en perspectiva cognitivista como la religiosidad cotidiana del creyente maduro. Para él la religiosidad madura es: "la predisposición a responder favorablemente, y de determinadas maneras habituales, a objetos o principios que el individuo considera como de última importancia en su vida, y como teniendo que ver con lo que él considera como permanente y central en la naturaleza de las cosas. ${ }^{39}$ Para Allport no es tan importante el contenido de la experiencia religiosa personal sino el significado global de la conducta religiosa de la persona. El tercer capítulo de Psicoanálisis y religión de Erich Fromm (1956) está dedicado al análisis de algunos tipos de experiencia religiosa. Fromm tampoco hace referencia a las experiencias sui generis sino que define la religión

36 JUNG, 1949, p. 9.

37 JUNG, Carl G. Respuesta a Job. Méjico: Fondo de Cultura Económica, 1964. p. 11.

38 MASLOW, 1994, p. 14.

39 ALLPORT, 1950, p. 63. 
como "cualquier sistema de pensamiento y acción compartido por un grupo, que dé al individuo una orientación y un objeto de devoción" .40 La religión es un hecho universal que radica en la naturaleza del hombre ya que según afirma "no existe nadie sin una necesidad religiosa, una necesidad de tener una orientación y un objeto de devoción" ${ }^{41}$ Su definición de religión es abierta y abarcativa. Para Frankl (1978) la religión es el significado último y está ligada a la búsqueda de sentido. La define citando las palabras del teólogo Paul Tillich: "ser religioso significa preguntarse apasionadamente por el sentido de nuestra existencia”. ${ }^{42}$ En esta línea no sui generis autores contemporáneos como Czachesz y Taves hacen referencia a otra concepción de la experiencia religiosa proveniente de las ciencias sociales que definen la religión y la experiencia religiosa a partir de categorías culturales. En psicología social, la teoría de la atribución busca explicar cómo los individuos utilizan la información que disponen para lograr entender y explicar las causas de comportamientos y eventos. Spilka y colaboradores la aplican a la comprensión de la experiencia religiosa. La consideran relacionada con la dimensión cognitiva del ser humano a través de la cual se le atribuye subjetivamente a determinada experiencia la cualidad de religiosa. Taves diferencia entre adscripción y atribución (la gente le adscribe calidad religiosa a una experiencia antes de atribuirle una causa religiosa) y sostiene que la experiencia religiosa no es un tipo especial de experiencia, sino una experiencia que el sujeto considera religiosa ("deemed religious").

\section{El aporte de las neurociencias}

Los nuevos aportes a la comprensión de la experiencia religiosa provienen del campo de las neurociencias, desde donde se busca entender lo que sucede en el cerebro de las personas cuando estas experimentan estados mentales que asocian con su fe religiosa. Los estudios de neurociencias se han enfocado principalmente en tres áreas: la relación entre la experiencia religiosa y las funciones cerebrales, entre la experiencia religiosa y la psicopatología, y entre la experiencia religiosa y diversas disciplinas o prácticas. Los estudios que investigan la relación de la experiencia religiosa con las funciones cerebrales utilizan en su mayoría tecnología de imagen funcional. Las pesquisas publicadas que utilizan esta tecnología en prácticas religiosas han sido numerosas durante los últimos años y han seguido diferentes líneas de investigación. También en el ámbito de las neurociencias se encuentran enfoques diversos y las diversas líneas de investigación siguen principalmente las dos concepciones antes mencionadas de la experiencia religiosa: la sui generis que tiene como uno de sus primeros y principales exponentes a Rudolf Otto, y la no sui generis. Para Eugene D'Aquili, Andrew Newberg, y Patrick McNamara entre otros, la experiencia religiosa es un tipo especial de experiencia, sui generis, mediada por estructuras cerebrales específicas. No es así para otros investigadores entre los que se

40 FROMM, 1956, p. 40.

41 FROMM, 1956, p. 44.

42 FRANKL, V. La presencia ignorada de Dios: psicoterapia y religión. Barcelona: Herder, 1978. p. 95. 
encuentran Nina Azari y Dieter Birnbacher,43 Uffe Schjoedt e István Czachesz. La investigación de la experiencia religiosa en este campo comporta varias dificultades. En primer lugar y como ya se ha visto, es un tema complejo, de difícil definición y ardua sistematización a causa de la amplitud de las variables. En segundo lugar, están los altos costos de las pesquisas y las limitaciones impuestas por la tecnología. También comportan dificultades la validez ecológica de los experimentos (las condiciones de los sujetos examinados durante los estudios son muy distintas de las naturales en las que viven sus prácticas religiosas); la implicación de los investigadores, sus elecciones metodológicas y su marco conceptual; y el riesgo de terceros de querer encontrar en los estudios respuestas ontológicas sobre las creencias y las experiencias religiosas.

\section{Reflexiones personales}

Al afrontar el estudio de la experiencia religiosa surge la necesidad de un abordaje interdisciplinar ya que se trata de afrontar una realidad compleja que toca todas las áreas del vivir humano. En el origen del recorrido realizado encontramos la descripción de las experiencias sui generis donde el aporte fenomenológico de Rudolf Otto ha abierto camino dejando una huella indeleble. Su concepción y descripción de la experiencia religiosa ejerció gran influencia, tanto que psicólogos relevantes como James, Jung y Maslow entre otros, se basaron en ella. También hoy, en el campo de las neurociencias experimentales, referentes importantes como Benson, ${ }^{44}$ d'Aquili y Newberg, 45 Previc y McNamara, ${ }^{46}$ la tienen como modelo al intentan encontrar un patrón de activación cerebral de estas experiencias. Junto a ellos hay otros investigadores que dan más importancia a los factores sociales y culturales. En esta línea Taves habla de la experiencia “considerada religiosa”, y

43 AZARI, N. P.; BIRNBACHER, D. The Role of Cognition and Feeling in Religious Experience. Zygon Journal of Religion and Science, v. 39, n. 4, p. 901-918, 2004; AZARI, N. P.; MISSIMER, J.; SEITZ, R. J. Religious Experience and Emotion: Evidence for Distinctive Cognitive Neural Patterns. The International Journal for the Psychology of Religion, v. 15, n. 4, p. 263-281, 2005; AZARI, N. P.; NICKEL, J.; WUNDERLICH, G.; NIEDEGGEN, M.; HEFTER, H.; TELLMANN, L.; SEITZ, R. J. Neural correlates of religious experience: Brain activity and religious experience. European Journal of Neuroscience, v. 13, n. 8, p. 1649-1652, 2001.

44 BENSON, H.; KLIPPER, M. Z. The Relaxation Response. Nueva York: William Morrow Paperbacks, 1975; BENSON, H.; MALHOTRA, M.; GOLDMAN, R. F.; JACOBS, G. D.; HOPKINS, J. Three Case Reports of the Metabolic and Electroencephalographic Changes during Advanced Buddhist Meditation Techniques. Behavioral Medicine, v. 16, n. 2, p. 90-95, 1990.

45 D'AQUILI, E. G.; NEWBERG, A. B. Religious and mystical states: a neuropsychological model. Zygon Journal of Religion and Science, v. 28, n. 2, p. 177-200, 1993; NEWBERG, A. B.; POURDEHNAD, M.; ALAVI, A.; BAIME, M. J.; SANTANNA, J.; D'AQUILI, E. G. The measurement of regional cerebral blood flow during the complex cognitive task of meditation: a preliminary SPECT study. Psychiatry Research: Neuroimaging, v. 106, n. 2, p. 113-122, 2001; NEWBERG, A. B.; POURDEHNAD, M.; ALAVI, A.; D'AQUILI, E. G. Cerebral blood flow during meditative prayer: preliminary findings and methodological issues. Perceptual and Motor Skills, v. 97, n. 2, p. 625-630, 2003.

46 McNAMARA, P. Where God and Science Meet. v. 1-3. Westport: Praeger, 2006; McNAMARA, P. The Neuroscience of Religious Experience. Nueva York: Cambridge University Press, 2009; WILDMAN, W.; McNAMARA, P. Challenges facing the neurological study of religious behavior, belief, and experience. Method and Theory in the Study of Religion, v. 20, p. 212-242, 2008. 
Azari y Birnbacher la estudian a partir de las emociones y dicen que la experiencia religiosa emerge como "un pensar que se siente como algo". El aporte de las neurociencias actualmente está dando lugar a lo que algunos autores están empezando a llamar Neuroteología. Las neurociencias experimentales tienen aún mucho que decir sobre la comprensión de la naturaleza de la experiencia religiosa y sobre el rol que esta tiene en la promoción de la salud y en el bienestar del individuo. La respuesta de relajación de Herbert Benson va en este sentido. Es importante notar también las dificultades que comporta trabajar en el campo. Además de los altos costos y de la necesidad de una mayor precisión y sensibilidad en los instrumentos de medición que se utilizan, hay dimensiones constitutivas de la experiencia que quedan fuera del alcance de estas investigaciones. Son por ejemplo la dimensión social y contextual de la experiencia religiosa ya que todos los estudios se realizan de forma individual y en laboratorios. Lo que sí es innegable, es la vigencia del aporte de Rudolf Otto a la comprensión de la experiencia religiosa.

\section{Referencias}

ALETTI, Mario. Percorsi di Psicologia della Religione alla luce della Psicoanalisi. 2.ed. Roma: Aracne, 2010.

ALLPORT, G. The individual and his religion: a psychological interpretation. London: Constable Publications, 1950.

.; ROSS, J. M. Personal religious orientation and prejudice. Journal of Personality and Social Psychology, v. 5, p. 432-443, 1967.

APUD, Ismael. Magia, ciencia y religión en antropología social. De Tylor a Levi-Strauss. Revista Crítica de Ciencias Sociales y Jurídicas, v. 30, n. 2, 2011.

ASOCIACIÓN PSICOLÓGICA AMERICANA. Asociación para la Psicología de la Religión y la Espiritualidad, División 36. Disponibel en: <http:/ / www.apa.org/about/division/div36.aspx>. Recuperado 4 feb. 2017.

AZARI, N. P.; BIRNBACHER, D. The Role of Cognition and Feeling in Religious Experience. Zygon Journal of Religion and Science, v. 39, n. 4, p. 901-918, 2004.

.; MISSIMER, J.; SEITZ, R. J. Religious Experience and Emotion: Evidence for Distinctive Cognitive Neural Patterns. The International Journal for the Psychology of Religion, v. 15, n. 4, p. 263-281, 2005.

.; NICKEL, J.; WUNDERLICH, G.; NIEDEGGEN, M.; HEFTER, H.; TELLMANN, L.; SEITZ, R. J. Neural correlates of religious experience: Brain activity and religious experience. European Journal of Neuroscience, v. 13, n. 8, p. 1649-1652, 2001.

BATSON, D. Religion and the Individual. New York: Oxford University Press, 1982.

BENSON, H.; KLIPPER, M. Z. The Relaxation Response. Nueva York: William Morrow Paperbacks, 1975. 
.; MALHOTRA, M.; GOLDMAN, R. F.; JACOBS, G. D.; HOPKINS, J. Three Case Reports of the Metabolic and Electroencephalographic Changes during Advanced Buddhist Meditation Techniques. Behavioral Medicine, v. 16, n. 2, p. 90-95, 1990.

CICERÓN. De natura deorum. Libro II, 28. Disponible en: <http:/ / www.progettovidio.it/dettagli1.asp?id=5519\&opera=De\%20Natura\%20Deorum \&libro=Libro\%20II>. Recuperado 5 feb. 2017.

CZACHESZ, I. Jesus' religious experience in the Gospels: toward a cognitive neuroscience approach. En: Jesus - Gestalt und Gestaltungen. Rezeptionen des Galiläers in Wissenschaft, Kirche und Gesellschaft. Göttingen: Vandenhoeck \& Ruprecht, 2013. p. 553-580.

. Tours of heaven in light of the neuroscientific study of religious experience. Journal of Cognitive Historiography, in press, v. 2, p. 33-55, 2015.

D'AQUILI, E. G.; NEWBERG, A. B. Religious and mystical states: a neuropsychological model. Zygon Journal of Religion and Science, v. 28, n. 2, p. 177-200, 1993.

DRU, A. The Journals of Søren Kierkegaard. Oxford: University Press, 1938.

ELIADE, M. Historia de las ideas y las creencias religiosas. v. I. Madrid: Ediciones Cristiandad, 1978.

FRANKL, V. La presencia ignorada de Dios: psicoterapia y religión. Barcelona: Herder, 1978.

FROMM, E. Psicoanálisis y religión. Buenos Aires: Editorial Psique, 1956.

HOOD, R.; SPILKA, B.; HILL, P. C. The Psychology of Religion: An empirical approach. 4.ed. New York: The Guilford Press, 1996.

JAMES, William. Las variedades de la experiencia religiosa: Un estudio de la naturaleza humana. Madrid: Península, 1994.

JUNG, Carl G. Psicología y religión. Buenos Aires: Paidós, 1949.

. Respuesta a Job. Méjico: Fondo de Cultura Económica, 1964.

Structure and dynamics of the psyche. v. 8. 2.ed. Princeton: Bollingen Series, 1969.

KANT, I. La religión dentro de los límites de la mera razón. Madrid: Alianza Editorial, 1969.

LACTANCIO. Divinae Institutiones. v. IV, 28. Madrid: Gredos, 1990.

LEWIS, C. T.; SHORT, C. Latin Dictionary. Oxford: Clarendon Press, 1879.

MASLOW, A. Religions, values and peak-experiences. London: Penguin Books, 1994.

McNAMARA, P. The Neuroscience of Religious Experience. Nueva York: Cambridge University Press, 2009.

. Where God and Science Meet. v. 1-3. Westport: Praeger, 2006. 
NEWBERG, A. B.; POURDEHNAD, M.; ALAVI, A.; BAIME, M. J.; SANTANNA, J.; D'AQUILI, E. G. The measurement of regional cerebral blood flow during the complex cognitive task of meditation: a preliminary SPECT study. Psychiatry Research: Neuroimaging, v. 106, n. 2, p. 113-122, 2001.

; .; D'AQUILI, E. G. Cerebral blood flow during meditative prayer: preliminary findings and methodological issues. Perceptual and Motor Skills, v. 97, n. 2, p. 625-630, 2003.

OTTO, R. Lo Santo: Lo racional y lo irracional en la idea de Dios. Madrid: Alianza Editorial, 2001.

REAL ACADEMIA ESPAÑOLA. Diccionario de la Lengua Española. 23.ed. Madrid: 2014. Disponible en: <http://www.rae.es/diccionario-de-la-lengua-espanola/la-23a-edicion2014>.

SCHJOEDT, U. The Religious Brain: A General Introduction to the Experimental Neuroscience of Religion. Method and Theory in the Study of Religion, v. 21, p. 310-339, 2009.

SPILKA, B.; SHAVER, P.; KIRKPATRICK, L. A. A general Attribution Theory for the Psychology of Religion. Journal for the Scientific Study of Religion, v. 24, n. 1, p. 1-20, 1985.

STEWART, D. J. The structure of the fihrist: Ibn Al-Nadim as historian of Islamic legal and theological schools. International Journal of Middle East Studies, v. 39, p. 369-387, 2007.

STRENSKI, I. The Shock of the «Savage»: Edward Burnett Tylor, Evolution, and Spirits. Thinking About Religion: An Historical Introduction to Theories of Religion. Oxford: Blackwell Publishing Ltd., 2006.

TAVES, A. Religious Experience. Encyclopedia of Religion. 2.ed. 2005. p. 7736-7750.

Religious Experience Reconsidered: A Building-Block Approach to the Study of Religion and Other Special Things. Princeton and Oxford: Princeton University Press, 2009.

THE GLOBAL RELIGIOUS LANDSCAPE. Pew Research Center. (2012). Disponible en: <http://www.pewforum.org/2012/12/18/global-religious-landscape-exec $>$. Recuperado 22 ene. 2017.

VELASCO, J. M. Introducción a la Fenomenología de la Religión. 7.ed. (corregida y ampliada). Madrid: Cristiandad, 1978.

. Religión. Disponible en: <ttp://www.mercaba.org/DicPC/R/religion.htm>. Recuperado 3 feb. 2017.

VERGOTE, Antoine. Psicología religiosa. Madrid: Taurus, 1966.

WILDMAN, W.; McNAMARA, P. Challenges facing the neurological study of religious behavior, belief, and experience. Method and Theory in the Study of Religion, v. 20, p. 212-242, 2008. 
YADEN, D.; EICHSTAEDT, J.; SCHWARTZ, A.; KERN, M.; NGUYEN, K. L.; WINTERING, N.; NEWBERG, A. The language of ineffability: linguistic analysis of mystical experiences. Psychology of Religion and Spirituality, 2015. 